\title{
Bouncing Cosmology for Entropy Corrected Models in Hořava-Lifshitz Gravity and Fractal Universe
}

\author{
Tanwi Bandyopadhyay * \\ Department of Mathematics, Adani Institute of Infrastructure Engineering, \\ Ahmedabad-382421, Gujarat, India. \\ Ujjal Debnath ${ }^{\dagger}$ \\ Department of Mathematics, Indian Institute of Engineering \\ Science and Technology, Shibpur, Howrah-711 103, India.
}

September 2, 2019

\begin{abstract}
The modified field equations are written in logarithmic and power law versions of entropy corrected models in Einstein's gravity in the background of FRW universe. In one section, a brief review of the Hořava-Lifshitz gravity is discussed and the modified field equations in logarithmic and power law versions of entropy corrected models in Hořava-Lifshitz gravity are formulated. The stability analysis for these models are performed by describing the dynamical system. In another section, a brief review of the fractal universe is presented and the modified field equations in logarithmic and power law versions of entropy corrected models in fractal universe are formulated. The stability analysis for the dynamical system for these models in the framework of fractal universe are described. Furthermore, the bouncing scenarios of the universe in Hořava-Lifshitz gravity and fractal model for both logarithmic and power law entropy corrected versions in $k=0,+1,-1$ separately are analyzed. For different cases, the validity of null energy condition (NEC) at the time of bounce is examined. Finally, the behaviors of the physical quantities are depicted through diagrams.
\end{abstract}

Keywords: Bouncing Cosmology, Entropy Corrected Models, Hořava-Lifshitz Gravity, Fractal Universe

\section{Contents}

1 Introduction $\quad 2$

2 Modified Equations for Entropy Corrected Models in Einstein's Gravity 3

2.1 Modified Equations from Logarithmic Corrected Entropy . . . . . . . . . . . . 3

2.2 Modified Equations from Power Law Corrected Entropy . . . . . . . . . . . . . . . 4

3 A Brief Review of Hořava-Lifshitz Gravity Theory

4 Dynamical System and Stability Analysis for Entropy Corrected Models in HořavaLifshitz Gravity

4.1 Logarithmic Corrected Model . . . . . . . . . . . . . . . . . . 5

4.2 Power Law Corrected Model . . . . . . . . . . . . . . . . . . 6

*tanwi.bandyopadhyay@aiim.ac.in

†ujjaldebnath@gmail.com 
6 Dynamical System and Stability Analysis for Entropy Corrected Models in Fractal Universe

6.1 Logarithmic Corrected Model . . . . . . . . . . . . . . . . . . . . . 9

6.2 Power Law Corrected Model . . . . . . . . . . . . . . . . . . . . . . . . 10

7 Connections Between Bounce and Energy Conditions 11

7.1 Logarithmic Corrected Model in HL Gravity . . . . . . . . . . . . . . . . . . . . . . . . 12

7.1 .1 Case I: $k=0 \ldots \ldots \ldots \ldots \ldots \ldots \ldots$

$7.1 .2 \quad$ Case II: $k= \pm 1 \ldots \ldots \ldots \ldots \ldots \ldots \ldots \ldots$

7.2 Power Law Corrected Model in HL Gravity . . . . . . . . . . . . . . . . . 13

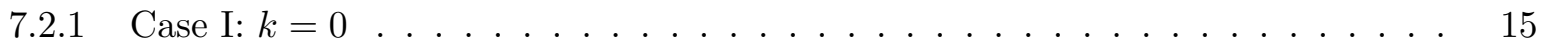

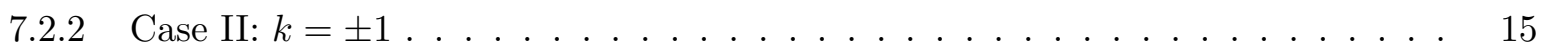

7.3 Logarithmic Corrected Model in Fractal Universe . . . . . . . . . . . . . . . . . . . 15

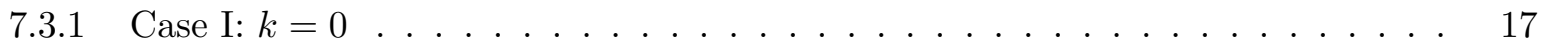

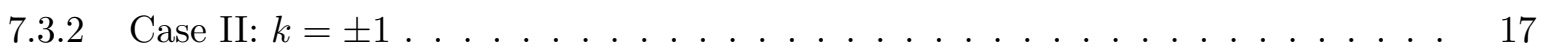

7.4 Power Law Corrected Model in Fractal Universe . . . . . . . . . . . . . . . 17

7.4 .1 Case I: $k=0 \ldots \ldots \ldots \ldots \ldots \ldots \ldots \ldots$

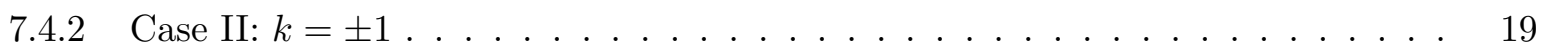

8 Discussions and Concluding Remarks $\quad 19$

\section{Introduction}

In the General Relativity (GR), the initial big bang singularity is the main problem in cosmological models [1]. Many theoretical inconsistencies have been occurred in the big bang cosmology. The consistent framework for this early time cosmology is the inflationary paradigm [2, 3, 4, 5]. However, the inflation theories suffer from the initial singularity problem at the origin $t=0[6]$. To resolve the initial singularity, the modification of GR theory [7] has been proposed (for example, Superstring theory, loop quantum gravity etc). To avoid the big bang singularity, an appealing alternative scenario is the big bounce scenario, where the singularity is replaced by a bounce [8, 9]. Another alternative to standard big bang cosmology (to avoid the big bang singularity) is oscillating universe where the big bang is replaced by cyclical evolution [10]. In the literatures, several authors have been studied the bouncing cosmology [11, 12, 13, 14, 15]. The bouncing cosmology has been studied in modified gravity theories eg. $f(R)$ gravity [16, 17, 18, 19, 20, 21, 22], $f(T)$ gravity [23, 24], loop quantum cosmology [25, 26, 27], braneworld gravity [28, 29, 30, 31], Brans-Dicke theory [32], Gauss-Bonnet gravity [33, 34, 35, 36]. Page [37] has discussed the fractal set of perpetually bouncing universes. Rip singularity scenario and bouncing universe in Chaplygin gas model have been studied in [38. Salehi [39] has studied the bouncing universe in presence of extended Chaplygin gas model. Also the study on the bouncing behavior of modified Chaplygin gas in presence of bulk viscosity has been discussed in [40]. Recently, we have studied [41] the bouncing scenarios of the universe in the framework of generalized cosmic Chaplygin gas and variable modified Chaplygin gas. In the literature, the bouncing nature of the universe in several kinds of dark energy models have been investigated [42, 43, 44, 45, 46]. Motivated by the above works, we investigate the bouncing cosmology for entropy corrected dark energy models in the framework of Hořava-Lifshitz gravity and fractal universe.

The organization of the paper is as follows: In section 2, we formulate the modified field equations in logarithmic and power law versions of entropy corrected models. In section 3, we briefly review the 
Hořava-Lifshitz gravity. In section 4, we write the modified field equations in logarithmic and power law versions of entropy corrected models in Hořava-Lifshitz gravity. Then we describe the dynamical system and stability analysis for these models. In section 5, we present a short review of the fractal universe. In section 6, we write the modified field equations in logarithmic and power law versions of entropy corrected models in fractal universe. Then we describe the dynamical system and stability analysis for these models in the framework of fractal universe. In section 7 , we study the bouncing scenarios of the universe in Hořava-Lifshitz gravity and fractal model for both logarithmic and power law entropy corrected versions. Finally, we present the conclusion in section 8 .

\section{Modified Equations for Entropy Corrected Models in Einstein's Gravity}

In the quantum field theory, the semi-classical quantum properties of black hole can be analyzed in curved backgrounds. Here matter is described by quantum field theory and gravity is described as a classical background. In this context, it is well known that the black hole can emit Hawking radiation where the temperature is proportional to its surface gravity on the black hole horizon and the entropy of the black hole is proportional to its horizon area. The black hole entropy $S$ plays an important role to study the astrophysical research, where $S=\frac{A}{4 G}$. Here $A$ is the area of the black hole horizon. In recent years, the quantum corrections (logarithmic and power law corrections) to the semi-classical entropy law have been introduced due to thermal equilibrium fluctuation or quantum fluctuation.

\subsection{Modified Equations from Logarithmic Corrected Entropy}

The logarithmic correction of entropy had been incorporated due to the thermal equilibrium or quantum fluctuations [47, 48, 49, 50, 51]. The Entropy-area relation in logarithmic correction of entropy is given by

$$
S=\frac{A}{4 G}+\alpha \ln \frac{A}{4 G}+\beta \frac{4 G}{A}
$$

where $\alpha, \beta$ are dimensionless constants of order unity.

We consider the Friedmann-Robertson-Walker (FRW) metric of the universe as

$$
d s^{2}=-d t^{2}+a^{2}(t)\left(\frac{d r^{2}}{1-k r^{2}}+r^{2}\left(d \theta^{2}+\sin ^{2} \theta d \phi^{2}\right)\right)
$$

where $k=(0, \pm 1)$ is the curvature scalar and $a(t)$ is the scale factor. Applying Clausius relation to apparent horizon of the FRW universe with any spatial curvature and using the above logarithmic corrected entropy area relation, Cai et al [52] found the modified field equations [52, 53] in Einstein's gravity as

$$
\left(H^{2}+\frac{k}{a^{2}}\right)+\frac{\alpha G}{2 \pi}\left(H^{2}+\frac{k}{a^{2}}\right)^{2}-\frac{\beta G^{2}}{3 \pi^{2}}\left(H^{2}+\frac{k}{a^{2}}\right)^{3}=\frac{8 \pi G}{3} \rho
$$

and

$$
\left[1+\frac{\alpha G}{\pi}\left(H^{2}+\frac{k}{a^{2}}\right)-\frac{\beta G^{2}}{2 \pi^{2}}\left(H^{2}+\frac{k}{a^{2}}\right)^{2}\right]\left(\dot{H}-\frac{k}{a^{2}}\right)=-4 \pi G(\rho+p)
$$

where $H=\frac{\dot{a}}{a}$ is the Hubble parameter. The bouncing scenario and stability analysis for modified equations in logarithmic corrected entropy has been investigated in [39]. 


\subsection{Modified Equations from Power Law Corrected Entropy}

From loop quantum gravity, the power law corrections arise due to the entanglement of quantum fields moving into and out of the horizon [54, 55, 56]. In this sense, the entropy-area relation for power law correction can be given as

$$
S=\frac{A}{4 G}\left[1-K_{\epsilon} A^{1-\frac{\epsilon}{2}}\right]
$$

where,

$$
K_{\epsilon}=\frac{\epsilon(4 \pi)^{\frac{\epsilon}{2}-1}}{(4-\epsilon) r_{c}^{2-\epsilon}}
$$

Here, $r_{c}$ is the crossover scale and $\epsilon$ is a dimensionless constant. By the similar procedure of Cai et al [52] and applying Clausius relation to apparent horizon of the FRW universe with a spatial curvature and using the above power law corrected entropy area relation, one can obtain the modified field equations for power law corrected entropy in Einstein's gravity in the following form

$$
\left(H^{2}+\frac{k}{a^{2}}\right)-\frac{1}{r_{c}^{2-\epsilon}}\left(H^{2}+\frac{k}{a^{2}}\right)^{\frac{\epsilon}{2}}=\frac{8 \pi G}{3} \rho
$$

and

$$
\left[2-\frac{\epsilon}{r_{c}^{2-\epsilon}}\left(H^{2}+\frac{k}{a^{2}}\right)^{\frac{\epsilon}{2}-1}\right]\left(\dot{H}-\frac{k}{a^{2}}\right)=-8 \pi G(\rho+p)
$$

\section{A Brief Review of Hořava-Lifshitz Gravity Theory}

Inspired by Lifshitz theory in solid state physics, Horava [57, 58, 59] proposed a UV complete field theory of gravity which is a non-relativistic renormalizable theory of gravity and reduces to Einstein's General Relativity at large scales, referred to the Horava-Lifshitz (HL) gravity theory. In the $(3+1)$ dimensional Arnowitt-Deser-Misner (ADM) formalism, the full metric can be written as 60, 61]

$$
d s^{2}=-N^{2} d t^{2}+g_{i j}\left(d x^{i}+N^{i} d t\right)\left(d x^{j}+N^{j} d t\right)
$$

Here the dynamical variables $N$ and $N_{i}$ are respectively the lapse and shift functions. Under the detailed balance condition, the full action for Hořava-Lifshitz gravity is given by [62

$$
\begin{gathered}
S=\int d t d^{3} x \sqrt{g} N\left[\frac{2}{\kappa^{2}}\left(K_{i j} K^{i j}-\lambda K^{2}\right)+\frac{\kappa^{2}}{2 \omega^{4}} C_{i j} C^{i j}-\frac{\kappa^{2} \mu \epsilon^{i j k}}{2 \omega^{2} \sqrt{g}} R_{i l} \nabla_{j} R_{k}^{l}\right. \\
\left.-\frac{\kappa^{2} \mu^{2}}{8} R_{i j} R^{i j}+\frac{\kappa^{2} \mu^{2}}{8(1-3 \lambda)}\left(\frac{1-4 \lambda}{4} R^{2}+\Lambda R-3 \Lambda^{2}\right)\right]
\end{gathered}
$$

where $K_{i j}$ is the extrinsic curvature taking the form:

$$
K_{i j}=\frac{1}{2 N}\left(\dot{g}_{i j}-\nabla_{i} N_{j}-\nabla_{j} N_{i}\right)
$$

and the Cotton tensor $C^{i j}$ takes the form:

$$
C^{i j}=\frac{\epsilon^{i k l}}{\sqrt{g}} \nabla_{k}\left(R_{i}^{j}-\frac{1}{4} R \delta_{l}^{j}\right)
$$


Here dot denotes a derivative with respect to $t$ and the covariant derivatives are defined with respect to the spatial metric $g_{i j}$. Here $\epsilon^{i j k}$ is the totally antisymmetric unit tensor, $\lambda$ is a dimensionless coupling constant and the parameters $\kappa, \omega$ and $\mu$ are constants with mass dimensions $-1,0,1$ respectively. Also $\Lambda$ is related to the cosmological constant $(>0)$ in the IR limit. Now, in the cosmology, the projectability condition is $N=1, N^{i}=0$. By varying $N$ and $g_{i j}$, the non-vanishing equations of motions can be expressed as

$$
H^{2}=\frac{\kappa^{2}}{6(3 \lambda-1)} \rho+\frac{\kappa^{2}}{6(3 \lambda-1)}\left[\frac{3 \kappa^{2} \mu^{2} k^{2}}{8(3 \lambda-1) a^{4}}+\frac{3 \kappa^{2} \mu^{2} \Lambda^{2}}{8(3 \lambda-1)}\right]-\frac{\kappa^{4} \mu^{2} k \Lambda}{8(3 \lambda-1)^{2} a^{2}}
$$

and

$$
\dot{H}+\frac{3}{2} H^{2}=-\frac{\kappa^{2}}{4(3 \lambda-1)} p-\frac{\kappa^{2}}{4(3 \lambda-1)}\left[\frac{\kappa^{2} \mu^{2} k^{2}}{8(3 \lambda-1) a^{4}}-\frac{3 \kappa^{2} \mu^{2} \Lambda^{2}}{8(3 \lambda-1)}\right]-\frac{\kappa^{4} \mu^{2} k \Lambda}{16(3 \lambda-1)^{2} a^{2}}
$$

let us assume

$$
G_{c}=\frac{\kappa^{2}}{16 \pi(3 \lambda-1)}, \quad \nu=\frac{\kappa^{4} \mu^{2} \Lambda}{8(3 \lambda-1)^{2}}>0
$$

where $G_{c}$ is the "cosmological" Newton's constant with $\lambda>1 / 3$. In Einstein's gravity, the Newton's gravitational constant is given by $G=\frac{\kappa^{2}}{32 \pi}$. So we can write $G_{c}=\frac{2 G}{3 \lambda-1}$. In fact, for $\lambda=1$, Lorentz invariance is restored $\left(G_{c}=G\right)$, while for $\lambda \neq 1$, Lorentz invariance is broken $\left(G_{c} \neq G\right)$. So we can express the above Friedmann equations in the following form:

$$
H^{2}+\frac{k \nu}{a^{2}}=\frac{8 \pi G_{c}}{3} \rho+\frac{k^{2} \nu}{2 \Lambda a^{4}}+\frac{\nu \Lambda}{2}
$$

and

$$
\dot{H}-\frac{k \nu}{a^{2}}=-4 \pi G_{c}(\rho+p)-\frac{k^{2} \nu}{\Lambda a^{4}}
$$

\section{Dynamical System and Stability Analysis for Entropy Corrected Models in Hořava-Lifshitz Gravity}

\subsection{Logarithmic Corrected Model}

By the similar procedure of Cai et al [52] and applying Clausius relation to apparent horizon of the FRW universe with a spatial curvature and using the logarithmic corrected entropy area relation, we can obtain the modified field equations in Hořava-Lifshitz (HL) gravity. The modified field equations for logarithmic corrected entropy will be the modifications of the equations (3), (4), (16) and (17), which are presented in the following form:

$$
\left(H^{2}+\frac{k \nu}{a^{2}}\right)+\frac{\alpha G_{c}}{2 \pi}\left(H^{2}+\frac{k}{a^{2}}\right)^{2}-\frac{\beta G_{c}^{2}}{3 \pi^{2}}\left(H^{2}+\frac{k}{a^{2}}\right)^{3}=\frac{8 \pi G_{c}}{3} \rho+\frac{k^{2} \nu}{2 \Lambda a^{4}}+\frac{\nu \Lambda}{2}
$$

and

$$
\left(\dot{H}-\frac{k \nu}{a^{2}}\right)+\left[\frac{\alpha G_{c}}{\pi}\left(H^{2}+\frac{k}{a^{2}}\right)-\frac{\beta G_{c}^{2}}{\pi^{2}}\left(H^{2}+\frac{k}{a^{2}}\right)^{2}\right]\left(\dot{H}-\frac{k}{a^{2}}\right)=-4 \pi G_{c}(\rho+p)-\frac{k^{2} \nu}{\Lambda a^{4}}
$$

The continuity equation is given by 


$$
\dot{\rho}+3 H(\rho+p)=0
$$

The study of dynamical system combined with phase space analysis is considered to be a strong tool to understand the behavior of the bouncing evolution of universe. It suggests that the analytical solutions for the model needs to be stable (or asymptotically stable under small perturbations). In the line with this idea, we introduce the following variables

$$
\chi=H, \quad \zeta=a, \quad \eta=\rho
$$

Then the field equations (18) and (19) reduce to the following autonomous system

$$
\dot{\chi}=\frac{-4 \pi G_{c}(1+\gamma) \eta-\frac{k^{2} \nu}{\Lambda \zeta^{4}}+\frac{k}{\zeta^{2}}\left[(1+\nu)+\alpha^{\prime}\left(\chi^{2}+\frac{k}{\zeta^{2}}\right)-\beta^{\prime}\left(\chi^{2}+\frac{k}{\zeta^{2}}\right)^{2}\right]}{1+\alpha^{\prime}\left(\chi^{2}+\frac{k}{\zeta^{2}}\right)-\beta^{\prime}\left(\chi^{2}+\frac{k}{\zeta^{2}}\right)^{2}}
$$

and

$$
\dot{\zeta}=\zeta \chi
$$

Here $\alpha^{\prime}=\frac{\alpha G_{c}}{\pi}$ and $\beta^{\prime}=\frac{\beta G_{c}{ }^{2}}{\pi^{2}}$. To find the critical points, we must have $\dot{\chi}=0, \dot{\zeta}=0$. Solving them, the critical point $\left(\chi_{c}, \zeta_{c}\right)$ can be obtained as

$$
\chi_{c}=0
$$

and $\zeta_{c}$ is the root of the equation

$$
\frac{3}{2}(1+\gamma) \nu \Lambda \zeta_{c}{ }^{6}-3(1+\gamma) k \nu \zeta_{c}{ }^{4}+\left[\frac{3}{2 \Lambda}(1+\gamma) k^{2} \nu+k(1+\nu)-(1+3 \gamma) \alpha^{\prime} k^{2}\right] \zeta_{c}{ }^{2}=\frac{(1+3 \gamma)}{2} \beta^{\prime} k^{3}
$$

It can be observed easily that the critical points depend upon a number of parameters. Thus one can have multiple analytical solutions for the critical points by fine tuning the parameters. Here we have obtained a stable critical point by assuming $k=-1, \Lambda=\alpha^{\prime}=\beta^{\prime}=1, \gamma=1 / 3, \nu=1$. The graph of $a$ vs $H$ in this case is shown in Fig.1 and we found the stable center.

\subsection{Power Law Corrected Model}

Similar to logarithmic corrected model in Hořava-Lifshitz gravity, the modified field equations for power law corrected entropy will be the modifications of the equations (7), (8), (16) and (17), which are presented in the following form:

$$
\left(H^{2}+\frac{k \nu}{a^{2}}\right)-\frac{1}{r_{c}^{2-\epsilon}}\left(H^{2}+\frac{k}{a^{2}}\right)^{\frac{\epsilon}{2}}=\frac{8 \pi G_{c}}{3} \rho+\frac{k^{2} \nu}{2 \Lambda a^{4}}+\frac{\nu \Lambda}{2}
$$

and

$$
2\left(\dot{H}-\frac{k \nu}{a^{2}}\right)-\frac{\epsilon}{r_{c}^{2-\epsilon}}\left(H^{2}+\frac{k}{a^{2}}\right)^{\frac{\epsilon}{2}-1}\left(\dot{H}-\frac{k}{a^{2}}\right)=-8 \pi G_{c}(\rho+p)-\frac{2 k^{2} \nu}{\Lambda a^{4}}
$$

Similar to the case of logarithmic corrected model in HL gravity, here we consider 


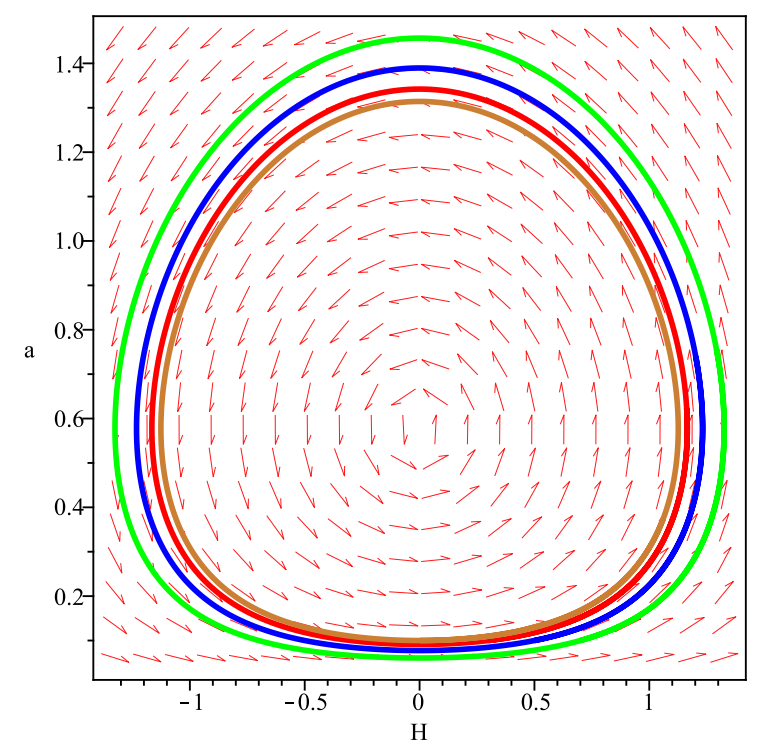

Fig.1

Fig.1: Phase diagram of $a$ vs $H$ in the Hor̆ava-Lifshitz gravity with logarithmic corrected model with the choice of the parameters as $k=-1, \Lambda=\alpha^{\prime}=\beta^{\prime}=1, \gamma=1 / 3, \nu=1$.

$$
\chi=H, \quad \zeta=a, \quad \eta=\rho
$$

Then the field equations (26) and (27) reduce to the following autonomous system

$$
\dot{\chi}=\frac{\frac{2 k \nu}{\zeta^{2}}-\frac{2 k^{2} \nu}{\Lambda \zeta^{4}}-3(1+\gamma)\left[\left(\chi^{2}+\frac{k \nu}{\zeta^{2}}\right)-\frac{1}{r_{c}^{2-\epsilon}}\left(\chi^{2}+\frac{k}{\zeta^{2}}\right)^{\frac{\epsilon}{2}}-\frac{k^{2} \nu}{2 \Lambda \zeta^{4}}-\frac{\nu \Lambda}{2}\right]-\frac{k \epsilon}{r_{c}^{2-\epsilon} \zeta^{2}}\left(\chi^{2}+\frac{k}{\zeta^{2}}\right)^{\frac{\epsilon}{2}-1}}{2-\frac{\epsilon}{r_{c}^{2-\epsilon}}\left(\chi^{2}+\frac{k}{\zeta^{2}}\right)^{\frac{\epsilon}{2}-1}}
$$

and

$$
\dot{\zeta}=\zeta \chi
$$

By putting $\dot{\chi}=0, \dot{\zeta}=0$, the critical point $\left(\chi_{c}, \zeta_{c}\right)$ can be obtained as

$$
\chi_{c}=0
$$

and $\zeta_{c}$ is the root of the equation

$$
\frac{\nu \Lambda}{2} \zeta_{c}{ }^{4-\epsilon}+\frac{k^{\frac{\epsilon}{2}}}{r_{c}{ }^{2-\epsilon}} \zeta_{c}^{3-\epsilon}+\frac{k^{2} \nu}{2 \Lambda} \zeta_{c}{ }^{-\epsilon}=\frac{\epsilon k^{\frac{\epsilon}{2}}}{3(1+\gamma) r_{c}{ }^{2-\epsilon}}
$$

In this case as well, the critical solutions depend on a number of parameters and hence more than one solution is possible by adjusting the parameters involved. We have found a stable critical point by choosing the parameters as $k=1, \Lambda=\nu=1, \epsilon=2, r_{c}=10$ and $\gamma=1 / 3$. The phase space 


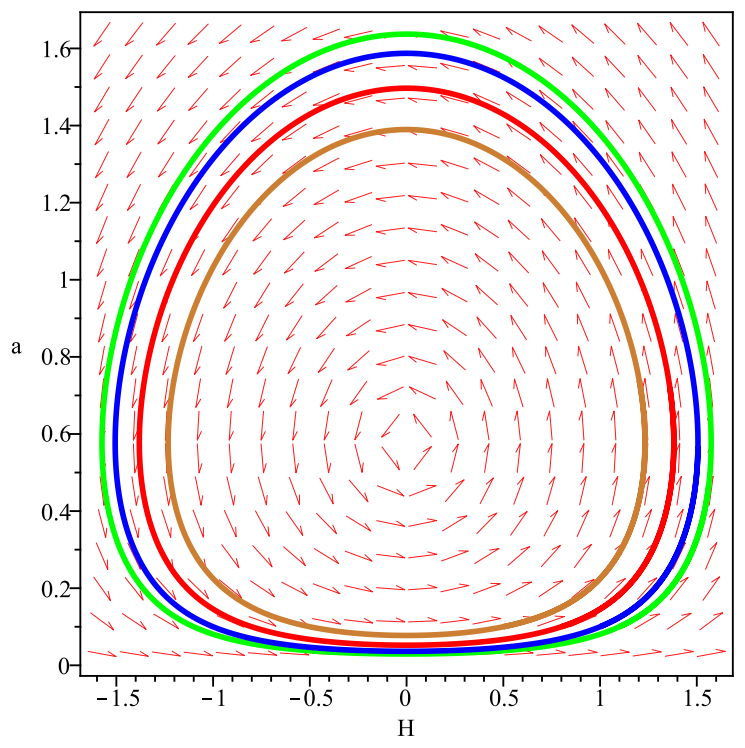

Fig.2

Fig.2: Phase diagram of $a$ vs $H$ in Hořava-Lifshitz gravity with power law corrected model with the choice of the parameters involved as $k=1, \Lambda=\nu=1, \epsilon=2, r_{c}=10$ and $\gamma=1 / 3$.

diagram of $a$ vs $H$ is shown in Fig.2 and we found the stable center.

\section{$5 \quad$ A Brief Review of Fractal Universe}

The idea of fractal effects in the Einstein's equations is one of the approach to cosmic acceleration in the gravity theory. Fractal features of quantum gravity and different cosmological properties in fractal universe have been discussed by Calcagni [63, 64]. In the Einstein's gravity, the action for the effective fractal space-time is given by 63 .

$$
S=\frac{1}{2 \kappa^{2}} \int\left(d^{4}(x)\right) v \sqrt{-g}\left(R-2 \Lambda-\omega \partial_{\mu} v \partial^{\mu} v+2 \kappa^{2} \mathcal{L}_{m}\right),
$$

where $g=\left|g_{\mu \nu}\right|, \kappa^{2}=8 \pi G, \Lambda$ is the cosmological constant, $R$ is the Ricci scalar, $\omega$ is the fractal parameter, $v$ is the fractal function and $\mathcal{L}_{m}$ is the matter Lagrangian. Taking the variation of the action given in (33), one can obtain the Friedmann equations in the fractal universe as [63]

$$
\left(H^{2}+\frac{k}{a^{2}}\right)+H \frac{\dot{v}}{v}-\frac{\omega}{3} \dot{v}^{2}=\frac{8 \pi G}{3} \rho+\frac{\Lambda}{3}
$$

and

$$
2\left(\dot{H}-\frac{k}{a^{2}}\right)-H \frac{\dot{v}}{v}+\omega \dot{v}^{2}+\frac{\ddot{v}}{v}=-8 \pi G(\rho+p)
$$

The continuity equation is given by 


$$
\dot{\rho}+\left(3 H+\frac{\dot{v}}{v}\right)(\rho+p)=0
$$

\section{Dynamical System and Stability Analysis for Entropy Corrected Models in Fractal Universe}

\subsection{Logarithmic Corrected Model}

Similar to logarithmic corrected model in Hořava-Lifshitz gravity, the modified field equations (modi-

fications of the equations (34), (35) ) for logarithmic corrected entropy in fractal Universe are obtained as

$$
\left(H^{2}+\frac{k}{a^{2}}\right)+\frac{\alpha G}{2 \pi}\left(H^{2}+\frac{k}{a^{2}}\right)^{2}-\frac{\beta G^{2}}{3 \pi^{2}}\left(H^{2}+\frac{k}{a^{2}}\right)^{3}+H \frac{\dot{v}}{v}-\frac{\omega}{3} \dot{v}^{2}=\frac{8 \pi G}{3} \rho+\frac{\Lambda}{3}
$$

and

$$
2\left[1+\frac{\alpha G}{\pi}\left(H^{2}+\frac{k}{a^{2}}\right)-\frac{\beta G^{2}}{\pi^{2}}\left(H^{2}+\frac{k}{a^{2}}\right)^{2}\right]\left(\dot{H}-\frac{k}{a^{2}}\right)-H \frac{\dot{v}}{v}+\omega \dot{v}^{2}+\frac{\ddot{v}}{v}=-8 \pi G(\rho+p)
$$

We follow the same procedure as followed in the previous two cases in Hořava-Lifshitz gravity. The variables are defined in the similar manner as

$$
\chi=H, \quad \zeta=a, \quad \eta=\rho
$$

Here we may choose $v=v_{0} a^{n}$, where $v_{0}$ and $n$ are constants. Then the modified field equations (37) and (38) would form the autonomous system given by

$$
\begin{gathered}
\dot{\chi}=\frac{1}{\left[1+\frac{n}{2}+\alpha^{\prime}\left(\chi^{2}+\frac{k}{\zeta^{2}}\right)-\beta^{\prime}\left(\chi^{2}+\frac{k}{\zeta^{2}}\right)^{2}\right]}\left\{(1+\gamma) \Lambda+\frac{2 k}{\zeta^{2}}\left[1+\alpha^{\prime}\left(\chi^{2}+\frac{k}{\zeta^{2}}\right)-\beta^{\prime}\left(\chi^{2}+\frac{k}{\zeta^{2}}\right)^{2}\right]\right. \\
-\chi^{2}\left(-n+n^{2}+\omega n^{2} v_{0}^{2} \zeta^{2 n}\right)-3(1+\gamma)\left[\left(\chi^{2}+\frac{k}{\zeta^{2}}\right)\left\{1+\frac{\alpha^{\prime}}{2}\left(\chi^{2}+\frac{k}{\zeta^{2}}\right)-\frac{\beta^{\prime}}{3}\left(\chi^{2}+\frac{k}{\zeta^{2}}\right)^{2}\right\}\right. \\
\left.\left.+\chi^{2}\left(n-\frac{1}{3} \omega n^{2} v_{0}^{2} \zeta^{2 n}\right)\right]\right\}
\end{gathered}
$$

and

$$
\dot{\zeta}=\zeta \chi
$$

Here $\alpha^{\prime}=\frac{\alpha G}{\pi}$ and $\beta^{\prime}=\frac{\beta G^{2}}{\pi^{2}}$. Solving the autonomous system, one can obtain the critical point $\left(\chi_{c}, \zeta_{c}\right)$ as

$$
\chi_{c}=0
$$

and $\zeta_{c}$ is the root of the equation 


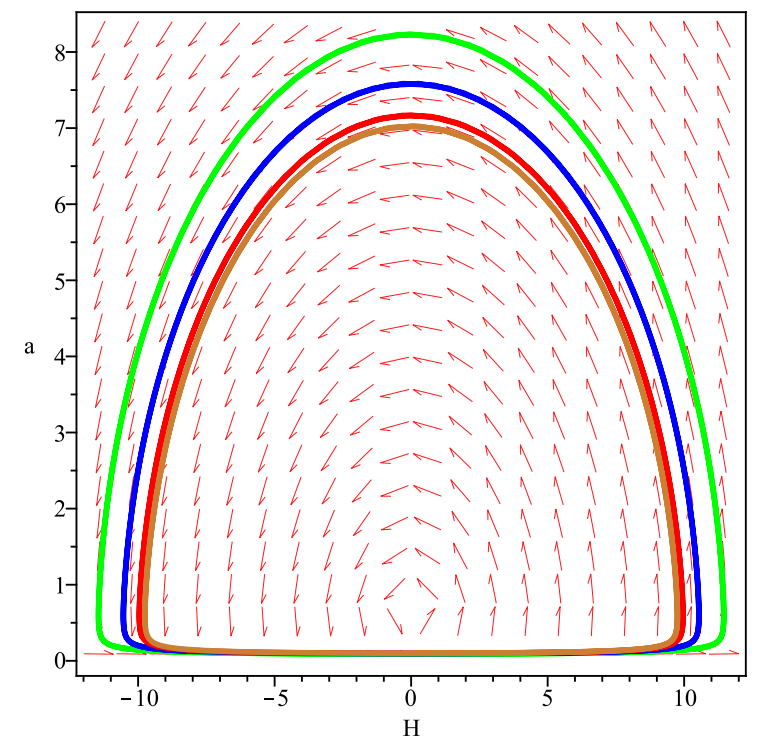

Fig.3

Fig. 3: Phase diagram of $a$ vs $H$ in fractal universe with logarithmic corrected model with the chosen values of the parameters involved as $k=1, \Lambda=\alpha^{\prime}=\beta^{\prime}=1, n=2, v_{0}=0.2, \omega=0.3$ and $\gamma=0$.

$$
(1+\gamma) \Lambda \zeta_{c}{ }^{6}-3(1+\gamma) k \zeta_{c}^{4}+\frac{(1-3 \gamma)}{2} k^{2} \alpha^{\prime} \zeta_{c}{ }^{2}+\beta^{\prime} \gamma k^{3}=0
$$

Again, the analytical expression of the evolving equation for $\zeta_{c}$ contains too many parameters. Hence we fine tune these parameters and present a stable critical point for the following choice of the parameters $k=1, \Lambda=\alpha^{\prime}=\beta^{\prime}=1, n=2, v_{0}=0.2, \omega=0.3$ and $\gamma=0$. The phase diagram for the stable center is drawn in Fig.3.

\subsection{Power Law Corrected Model}

Similar to power law corrected model in Hořava-Lifshitz gravity, the modified field equations (modifications of the equations (34), (35) ) for power law corrected entropy in fractal Universe are obtained as

$$
\left(H^{2}+\frac{k}{a^{2}}\right)-\frac{1}{r_{c}^{2-\epsilon}}\left(H^{2}+\frac{k}{a^{2}}\right)^{\frac{\epsilon}{2}}+H \frac{\dot{v}}{v}-\frac{\omega}{3} \dot{v}^{2}=\frac{8 \pi G}{3} \rho+\frac{\Lambda}{3}
$$

and

$$
\left[2-\frac{\epsilon}{r_{c}^{2-\epsilon}}\left(H^{2}+\frac{k}{a^{2}}\right)^{\frac{\epsilon}{2}-1}\right]\left(\dot{H}-\frac{k}{a^{2}}\right)-H \frac{\dot{v}}{v}+\omega \dot{v}^{2}+\frac{\ddot{v}}{v}=-8 \pi G(\rho+p)
$$

A similar procedure is carried out with the variables defined as 


$$
\chi=H, \quad \zeta=a, \quad \eta=\rho
$$

Then the modified field equations (44) and (45) reduce to the following autonomous system

$$
\begin{gathered}
\dot{\chi}=\frac{1}{\left[2+n-\frac{\epsilon}{r_{c}^{2-\epsilon}}\left(\chi^{2}+\frac{k}{\zeta^{2}}\right)^{\frac{\epsilon}{2}-1}\right]}\left[\frac{2 k}{\zeta^{2}}-\frac{k \epsilon}{\zeta^{2} r_{c}^{2-\epsilon}}\left(\chi^{2}+\frac{k}{\zeta^{2}}\right)^{\frac{\epsilon}{2}-1}\right. \\
\left.+\chi^{2}\left(n-n^{2}-n^{2} \omega v_{0}^{2} \zeta^{2 n}\right)-3(1+\gamma)\left\{\frac{k}{\zeta^{2}}-\frac{\Lambda}{3}+\chi^{2}\left(1+n-\frac{\omega}{3} n^{2} v_{0}^{2} \zeta^{2 n}\right)-\frac{1}{r_{c}^{2-\epsilon}}\left(\chi^{2}+\frac{k}{\zeta^{2}}\right)^{\frac{\epsilon}{2}}\right\}\right]
\end{gathered}
$$

and

$$
\dot{\zeta}=\zeta \chi
$$

The critical point $\left(\chi_{c}, \zeta_{c}\right)$ can be obtained by solving the above equations as

$$
\chi_{c}=0
$$

and $\zeta_{c}$, beings the root of the equation

$$
(3+3 \gamma-\epsilon) k^{\frac{\epsilon}{2}}\left(\frac{\zeta_{c}}{r_{c}}\right)^{2-\epsilon}=k(1+3 \gamma)-\Lambda(1+\gamma) \zeta_{c}^{2}
$$

Again we choose particular values to the various parameters in order to attain a stable critical point. The values are $k=-1, \Lambda=1, \epsilon=4, r_{c}=10, n=2, v_{0}=0.5, \omega=0.6$ and $\gamma=-1 / 2$. The phase space diagram of $a$ vs $H$ is presented in Fig.4 and we found the stable center.

\section{Connections Between Bounce and Energy Conditions}

To avoid the initial singularity problem of General Relativity, many attempts had been made by proposing different modified or generalized theories of gravity. One of these is the non-singular bouncing cosmological model. By modifying the action, one can achieve an oscillating universe with an initial narrow state by a minimal radius, which further develops to an expanding phase. This implies that the universe arrives into the Big Bang era after the bounce happens, thus avoids the initial singularity and results in a cyclic evolution. In terms of the scale factor, one can make the argument as follows. During the initial state of bounce, the scale factor decreases $(\dot{a}(t)<0)$ and the universe gets contracted. At the time of 'bounce' $\left(t=t_{b}\right.$, say), the scale factor attains a minimum. The criteria for this is $\dot{a}(t)=0$ and $\ddot{a}(t)>0$ for $t \in\left(t_{b}-\epsilon, t_{b}\right) \cup\left(t_{b}, t_{b}+\epsilon\right)$ for small $\epsilon>0$. After the bounce, the scale factor increases again $(\dot{a}(t)>0)$ and subsequently the universe undergoes an expanding phase. One must note that for a non-singular bounce, $a\left(t_{b}\right) \neq 0$. However, the conditions may not be sufficient.

In this section, we investigate the bouncing solutions of the present models in the light of energy conditions. Our main focus is to verify the Null Energy Condition (NEC) $(\rho+p \geq 0)$ as violation of this condition automatically implies the violation of all other point-wise energy conditions. 


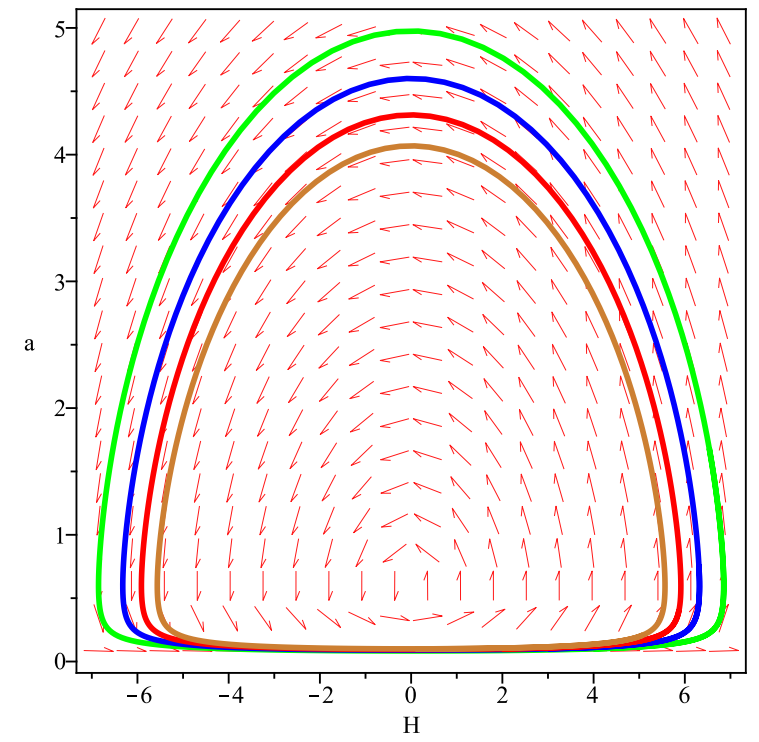

Fig.4

Fig. 4: Phase diagram of $a$ vs $H$ in fractal universe with power law corrected model with the chosen values of the parameters involved as $k=-1, \Lambda=1, \epsilon=4, r_{c}=10, n=2, v_{0}=0.5, \omega=0.6$ and $\gamma=-1 / 2$.

\subsection{Logarithmic Corrected Model in HL Gravity}

Here we use (19) during bounce and have

$$
4 \pi G_{c}\left(\rho_{b}+p_{b}\right)=\left(\frac{k \nu}{a_{b}{ }^{2}}+\frac{\alpha^{\prime} k^{2}}{a_{b}{ }^{4}}-\frac{k^{2} \nu}{\Lambda a_{b}{ }^{4}}\right)-\frac{\ddot{a}_{b}}{a_{b}}\left(1+\frac{\alpha^{\prime} k}{a_{b}{ }^{2}}-\frac{\beta^{\prime} k^{2}}{a_{b}{ }^{4}}\right)
$$

Here, $\rho_{b}, p_{b}$ and $a_{b}$ signify the energy density, pressure and the scale factor during bounce, i.e., at $t=t_{b}$. The right hand side of the above equation must be positive for NEC to be satisfied. The energy density at bounce in this case is given by

$$
\rho_{b}=\frac{3}{8 \pi G_{c}}\left[\frac{k \nu}{a_{b}{ }^{2}}+\frac{\alpha^{\prime} k^{2}}{a_{b}{ }^{4}}-\frac{k^{2} \nu}{2 \Lambda a_{b}{ }^{4}}-\frac{\beta^{\prime} k^{3}}{3 a_{b}{ }^{6}}-\frac{\nu \Lambda}{2}\right]
$$

At the bounce, the continuity equation

$$
\dot{\rho}=-3 H(\rho+p)
$$

would become

$$
\dot{\rho}_{b}=-3 H_{b}\left(\rho_{b}+p_{b}\right)
$$

As we know that $H_{b}=\frac{a_{b}}{a_{b}}=0$, hence $\dot{\rho}_{b}=0$ and consequently $\rho_{b}$ reaches an extremum energy density at bounce. Further

$$
\ddot{\rho}_{b}=-3 \dot{H}_{b}\left(\rho_{b}+p_{b}\right)
$$


Setting different values of $k$, we can find the restrictions for the satisfaction of NEC and consequently the extremum energy density during bounce.

\subsubsection{Case I: $k=0$}

From (51), we get

$$
\left(\rho_{b}+p_{b}\right)=-\frac{1}{4 \pi G_{c}}\left(\frac{\ddot{a_{b}}}{a_{b}}\right)<0, \text { since } \ddot{a}_{b}>0
$$

It can be seen that the NEC is thus being violated. Also we see that $\ddot{\rho}_{b}>0$. Therefore $\rho_{b}$ must be attaining a minimum value during bounce given by $\left(\rho_{b}\right)_{\min }=-\frac{3 \nu \Lambda}{16 \pi G_{c}}$. This case therefore turns out to be unfavorable for bounce.

\subsubsection{Case II: $k= \pm 1$}

From (55) we can say that in order to satisfy the NEC, we must have $\ddot{\rho}_{b}<0$ as $\dot{H}_{b}>0$ during bounce. Thus $\rho_{b}$ in both the cases of $k=1$ and $k=-1$ attains a maximum value given by

$$
\begin{gathered}
\rho_{\left.b_{\text {max }}\right|_{k=1}}=\frac{3}{8 \pi G_{c}}\left[\frac{\nu}{a_{b}{ }^{2}}+\left(\alpha^{\prime}-\frac{\nu}{2 \Lambda}\right) \frac{1}{a_{b}{ }^{4}}-\frac{\beta^{\prime}}{3 a_{b}{ }^{6}}-\frac{\nu \Lambda}{2}\right] \\
\rho_{\left.b_{\max }\right|_{k=-1}}=\frac{3}{8 \pi G_{c}}\left[-\frac{\nu}{a_{b}{ }^{2}}+\left(\alpha^{\prime}-\frac{\nu}{2 \Lambda}\right) \frac{1}{a_{b}{ }^{4}}+\frac{\beta^{\prime}}{3 a_{b}{ }^{6}}-\frac{\nu \Lambda}{2}\right]
\end{gathered}
$$

The criteria for the satisfaction of NEC for the two cases are

$$
\begin{gathered}
\frac{\alpha^{\prime}}{a_{b}^{2}}+\nu\left(1-\frac{1}{\Lambda a_{b}^{2}}\right)>a_{b} \ddot{a}_{b}\left(1+\frac{\alpha^{\prime}}{a_{b}^{2}}-\frac{\beta^{\prime}}{a_{b}^{4}}\right), \quad k=1 \\
\frac{\alpha^{\prime}}{a_{b}^{2}}-\nu\left(1+\frac{1}{\Lambda a_{b}^{2}}\right)>a_{b} \ddot{a}_{b}\left(1-\frac{\alpha^{\prime}}{a_{b}^{2}}-\frac{\beta^{\prime}}{a_{b}^{4}}\right), \quad k=-1
\end{gathered}
$$

The evolution of the scale factor $a$, the sum of the energy density and pressure $(\rho+p)$ and the energy density $\rho$ during the bounce period for logarithmic corrected universe in HL gravity are shown in Figs. 5-7. Variations in the initial conditions result in different colored plots. A barotropic equation of state $p=\gamma \rho$ is assumed for the matter content. For viable choices of the parameters involved, the plots show an oscillating universe with the NEC being satisfied.

\subsection{Power Law Corrected Model in HL Gravity}

From (27) we have

$$
8 \pi G_{c}\left(\rho_{b}+p_{b}\right)=\left[\frac{2 k \nu}{a_{b}^{2}}\left(1-\frac{k}{\Lambda a_{b}^{2}}\right)-\frac{\epsilon k^{\frac{\epsilon}{2}}}{a_{b} r_{c}^{2-\epsilon}}\right]-\frac{\ddot{a_{b}}}{a_{b}}\left[2-\frac{\epsilon}{r_{c}^{2-\epsilon}}\left(\frac{k}{a_{b}^{2}}\right)^{\frac{\epsilon}{2}-1}\right]
$$

For the NEC to be satisfied, the right hand side of the above equation needs to be positive. The energy density at the time of bounce in this case is given by

$$
\rho_{b}=\frac{3}{8 \pi G_{c}}\left[\frac{k \nu}{a_{b}^{2}}-\frac{1}{r_{c}^{2-\epsilon}}\left(\frac{k}{a_{b}^{2}}\right)^{\frac{\epsilon}{2}}-\frac{k^{2} \nu}{2 \Lambda a_{b}^{4}}-\frac{\nu \Lambda}{2}\right]
$$




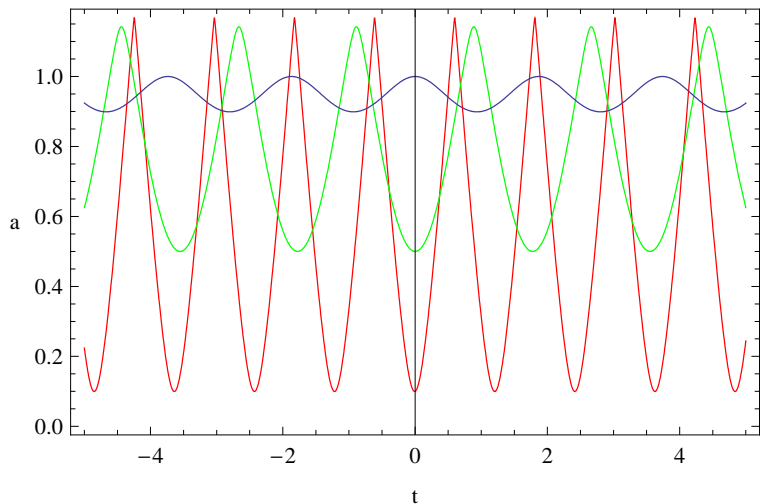

Fig.5

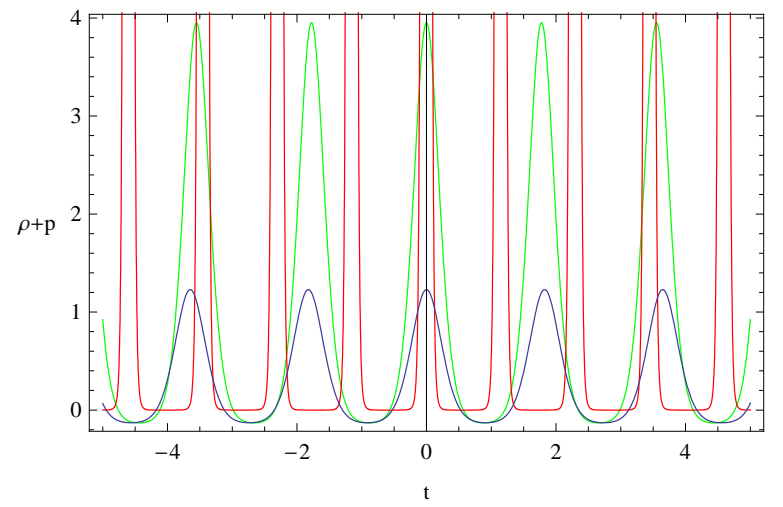

Fig.6

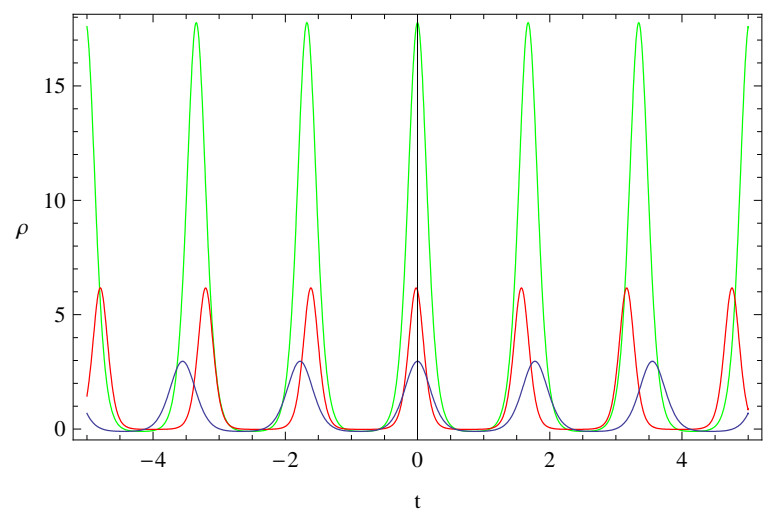

Fig.7

Figs. 5-7 show the dynamical behaviors of the scale factor $a$, the sum of the energy density and pressure $(\rho+p)$ and the energy density $\rho$ during the bounce period for logarithmic corrected universe in HL gravity. The parameters are chosen for this case as $k=-1, G_{c}=\alpha^{\prime}=\beta^{\prime}=\nu=\Lambda=1$ and $\gamma=1 / 3$. Different colors signify different initial conditions. 


\subsubsection{Case I: $k=0$}

This case is identical to the subsection 7.1.1. Here also the NEC is violated and at the time of bounce, the energy density becomes negative concluding no bounce.

\subsubsection{Case II: $k= \pm 1$}

From (55) we can say that in order to satisfy the NEC, we get $\ddot{\rho}_{b}<0$. Thus $\rho_{b}$ in both the cases of $k=1$ and $k=-1$ attains a maximum value given by

$$
\begin{gathered}
\left.\rho_{b \max }\right|_{k=1}=\frac{3}{8 \pi G_{c}}\left[\frac{\nu}{a_{b}{ }^{2}}-\frac{\nu}{2 \Lambda a_{b}{ }^{4}}-\frac{\nu \Lambda}{2}-\frac{1}{a_{b}{ }^{\epsilon} r_{c}{ }^{2-\epsilon}}\right] \\
\rho_{\left.b_{\max }\right|_{k=-1}}=-\frac{3}{8 \pi G_{c}}\left[\frac{\nu}{a_{b}{ }^{2}}+\frac{\nu}{2 \Lambda a_{b}{ }^{4}}+\frac{\nu \Lambda}{2}+\frac{(-1)^{\frac{\epsilon}{2}}}{a_{b}{ }^{\epsilon} r_{c}{ }^{2-\epsilon}}\right]
\end{gathered}
$$

It is interesting to note that the fourth term of (64) is the deciding term for a feasible $\rho_{b}$ during bounce. For $\epsilon=2 m$, where $m$ is odd, there would be a chance to have a positive $\rho_{b}$. In all other cases, $\rho_{b}$ will be negative concluding unfavorable condition for bounce. The criteria for the satisfaction of NEC for $k=1$ and $k=-1$ will be

$$
\begin{gathered}
\frac{2 \nu}{a_{b}}\left(1-\frac{1}{\Lambda a_{b}^{2}}\right)-\frac{\epsilon}{r_{c}^{2-\epsilon}}>\ddot{a_{b}}\left[2-\frac{\epsilon}{\left(a_{b} / r_{c}\right)^{\epsilon-2}}\right], \quad k=1 \\
\frac{2 \nu}{a_{b}}\left(1+\frac{1}{\Lambda a_{b}^{2}}\right)+\frac{\epsilon(-1)^{\frac{\epsilon}{2}}}{r_{c}^{2-\epsilon}}+\ddot{a_{b}}\left[2+\frac{\epsilon(-1)^{\frac{\epsilon}{2}}}{\left(a_{b} / r_{c}\right)^{\epsilon-2}}\right]>0, \quad k=-1
\end{gathered}
$$

The evolution of the scale factor $a$, the sum of the energy density and pressure $(\rho+p)$ and the energy density $\rho$ during the bounce period for power law corrected universe in HL gravity are shown in Figs. 8-10. A barotropic equation of state $p=\gamma \rho$ is assumed for the matter content. Different sets of parameters have been chosen to generate the plots. For scale factor, the parameters involved are chosen as $k=\epsilon=\nu=\Lambda=G_{c}=1$ and $r_{c}=10$ to observe a bouncing solution in this model. Here the variation is considered in the initial conditions which produces different colored plots. On the other hand, to generate the plots of $(\rho+p)$ and $\rho$, the choice of $\epsilon$ is varied since this plays a major role in defining the satisfaction of the NEC. Here we chose $k=-1, \nu=\Lambda=G_{c}=1, r_{c}=10$ and $\epsilon=6$ (green line), 10 (red line) and 14 (blue line). it can be observed that the plots show the satisfaction of the NEC.

\subsection{Logarithmic Corrected Model in Fractal Universe}

From (38) we get

$$
8 \pi G\left(\rho_{b}+p_{b}\right)=\frac{2 k^{2}}{a_{b}{ }^{4}}\left(1+\alpha^{\prime}-\frac{\beta^{\prime} k}{a_{b}^{2}}\right)-\frac{\ddot{a_{b}}}{a_{b}}\left(n+\frac{2 k \alpha^{\prime}}{a_{b}{ }^{2}}\right)
$$

where the energy density at the time of bounce in this case is given by

$$
\rho_{b}=\frac{3}{8 \pi G}\left[\frac{k}{a_{b}{ }^{2}}+\frac{k^{2} \alpha^{\prime}}{a_{b}{ }^{4}}-\frac{k^{3} \beta^{\prime}}{3 a_{b}{ }^{6}}-\frac{\Lambda}{3}\right]
$$

From the continuity equation (36), we can write

$$
\dot{\rho_{b}}=-(n+3) H_{b}\left(\rho_{b}+p_{b}\right)
$$




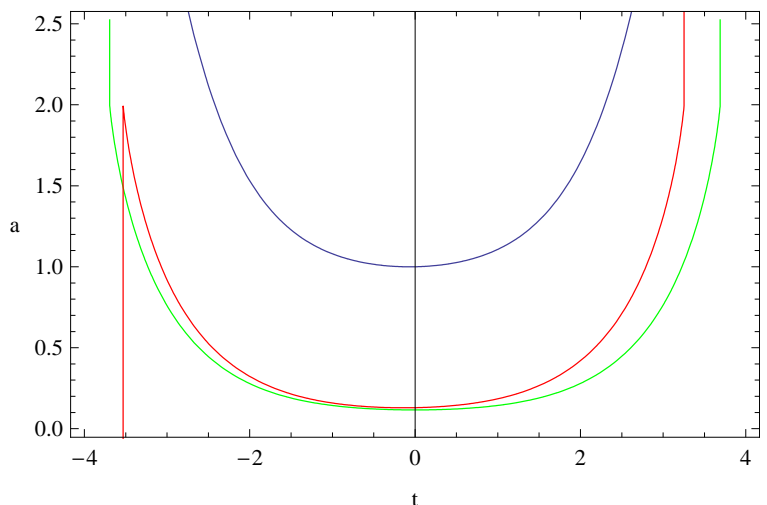

Fig.8

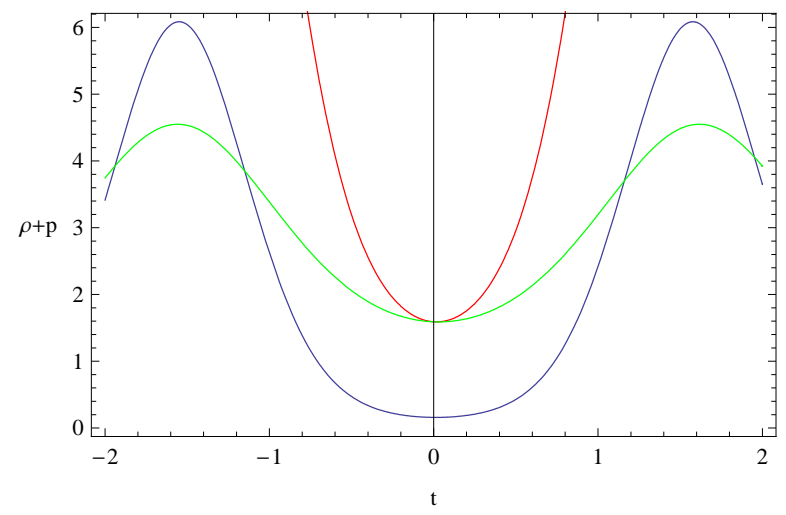

Fig.9

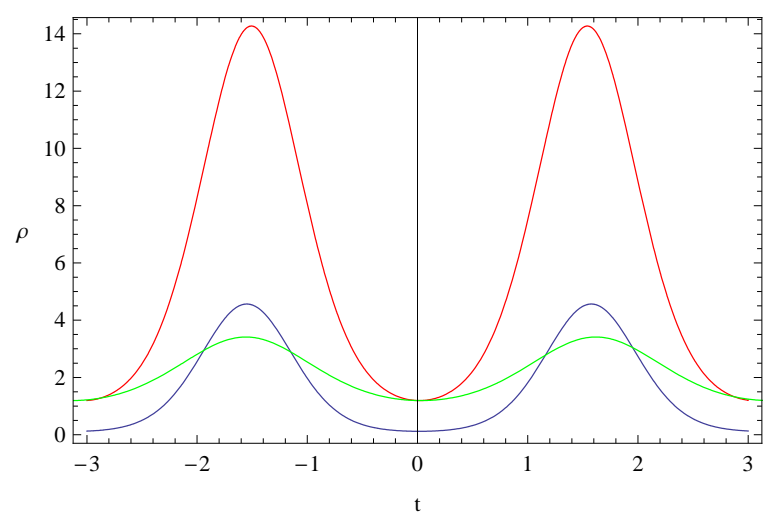

Fig.10

Figs. 8-10 show the dynamical behaviors of the scale factor $a$, the sum of the energy density and pressure $(\rho+p)$ and the energy density $\rho$ during the bounce period for power law corrected universe in HL gravity. 
Again as $H_{b}=0$, we have $\dot{\rho}_{b}=0$ and subsequently $\rho_{b}$ attains a local extremum during bounce. Further

$$
\ddot{\rho}_{b}=-(n+3) \dot{H}_{b}\left(\rho_{b}+p_{b}\right)
$$

Assuming the validity of NEC, we therefore would have $\ddot{\rho}_{b}<0$ if $n>-3$ implying a local maximum value for $\rho_{b}$ and otherwise a local minimum value for $\rho_{b}$.

\subsubsection{Case I: $k=0$}

In this case we have

$$
\rho_{b}+p_{b}=-\frac{n}{8 \pi G} \frac{\ddot{a}_{b}}{a_{b}}
$$

It is evident that the NEC is satisfied for a negative $n$ and violated otherwise. The energy density at bounce is given by $\rho_{b}=-\frac{\Lambda}{8 \pi G}<0$. Thus this case is also not favorable for bounce.

\subsubsection{Case II: $k= \pm 1$}

In this case the criteria for the satisfaction of NEC are given by

$$
2\left(1+\alpha^{\prime}-\frac{\beta^{\prime}}{a_{b}^{2}}\right)>a_{b} \ddot{a_{b}}\left(n+\frac{2 \alpha^{\prime}}{a_{b}^{2}}\right), \quad k=1
$$

and

$$
2\left(1+\alpha^{\prime}+\frac{\beta^{\prime}}{a_{b}^{2}}\right)>a_{b} \ddot{a_{b}}\left(n-\frac{2 \alpha^{\prime}}{a_{b}^{2}}\right), \quad k=-1
$$

The evolution of the scale factor $a$, the sum of the energy density and pressure $(\rho+p)$ and the energy density $\rho$ during the bounce period for logarithmic corrected model in fractal universe are shown in Figs. 11-13. A barotropic equation of state $p=\gamma \rho$ is assumed for the matter content. Here the initial conditions are kept constant but the choice of $\gamma$ is varied. For viable choices of the parameters involved, the plots clearly show a bouncing solution with the NEC being satisfied.

\subsection{Power Law Corrected Model in Fractal Universe}

In this case, we get from (45)

$$
8 \pi G\left(\rho_{b}+p_{b}\right)=\frac{k}{a_{b}^{2}}\left[2-\frac{\epsilon k^{\frac{\epsilon}{2}-1}}{\left(a_{b} / r_{c}\right)^{\epsilon-2}}\right]-\frac{\ddot{a_{b}}}{a_{b}}\left[(n+2)-\frac{\epsilon k^{\frac{\epsilon}{2}-1}}{\left(a_{b} / r_{c}\right)^{\epsilon-2}}\right]
$$

The right hand side of the above expression must be positive for the validity of the NEC. Here the energy density during bounce is given by

$$
\rho_{b}=\frac{3}{8 \pi G}\left[\frac{k}{a_{b}^{2}}-\frac{\Lambda}{3}-\frac{k^{\frac{\epsilon}{2}}}{a_{b}{ }^{\epsilon} r_{c}^{2-\epsilon}}\right]
$$




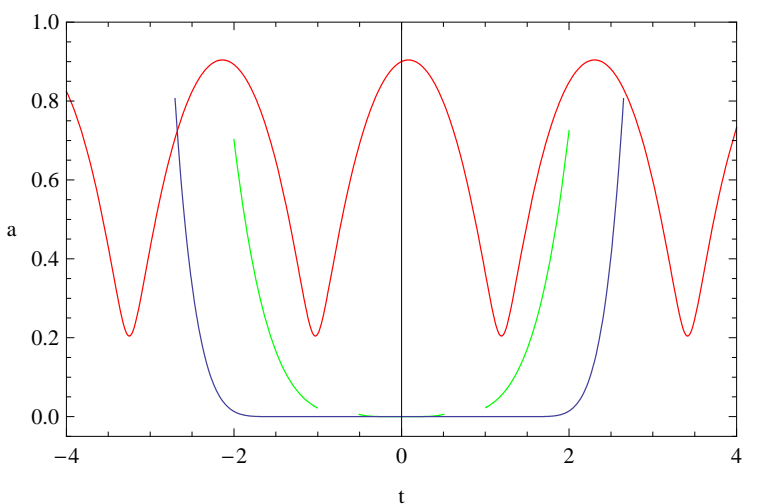

Fig.11

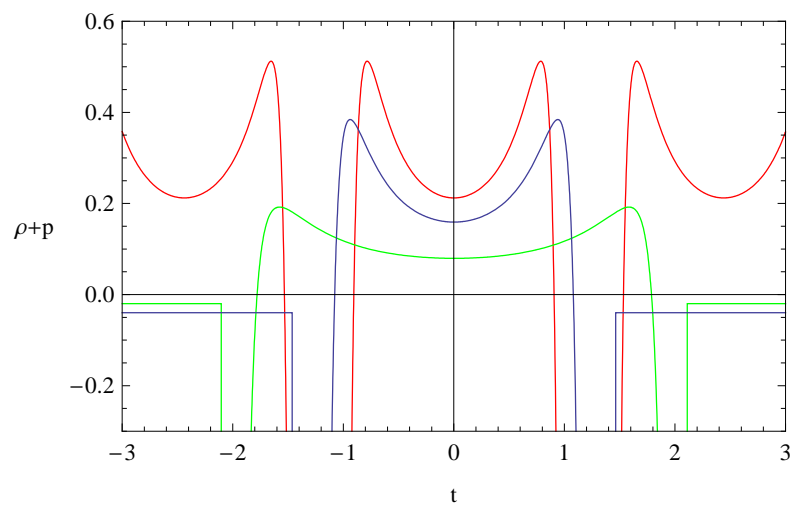

Fig.12

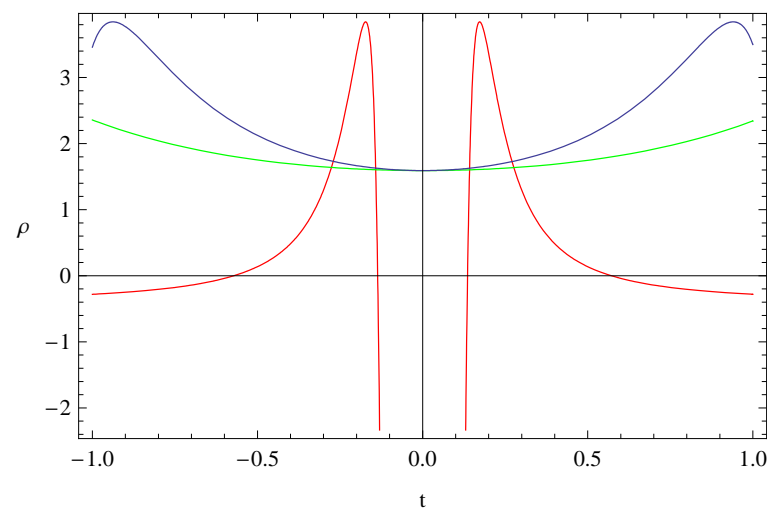

Fig.13

Figs. 11-13 show the dynamical behaviors of the scale factor $a$, the sum of the energy density and pressure $(\rho+p)$ and the energy density $\rho$ during the bounce period for logarithmic corrected model in fractal universe. The parameters are chosen for this case as $k=G=\alpha^{\prime}=\beta^{\prime}=\Lambda=1$ and $n=v_{0}=2$ and $\gamma=0$ (blue line), $1 / 3$ (red line) and $-1 / 2$ (green line). 


\subsubsection{Case I: $k=0$}

Here

$$
\rho_{b}+p_{b}=-\frac{(n+2)}{8 \pi G} \frac{\ddot{a}_{b}}{a_{b}}
$$

This means that NEC is satisfied when $n<-2$. In this case $\rho_{b}=-\frac{\Lambda}{3 \pi G}$ implying a solution without favorable criteria for a bounce.

\subsubsection{Case II: $k= \pm 1$}

The energy density in this case is given by

$$
\rho_{\left.b\right|_{k=1}}=\frac{3}{8 \pi G}\left[\frac{1}{a_{b}^{2}}-\frac{\Lambda}{3}-\frac{1}{a_{b}{ }^{\epsilon} r_{c}^{2-\epsilon}}\right]
$$

and

$$
\rho_{\left.b\right|_{k=-1}}=-\frac{3}{8 \pi G}\left[\frac{1}{a_{b}{ }^{2}}+\frac{\Lambda}{3}+\frac{(-1)^{\frac{\epsilon}{2}}}{a_{b}{ }^{\epsilon} r_{c}^{2-\epsilon}}\right]
$$

It may be noted that the third term decides for a non negative $\rho_{b}$. If $\epsilon=2 m$, where $m$ is odd, then one can attain a bouncing solution. Otherwise there will be no bounce.

The criteria for the validity of the NEC in the two cases $(k= \pm 1)$ are

$$
\begin{gathered}
2-\frac{\epsilon}{\left(a_{b} / r_{c}\right)^{\epsilon-2}}>a_{b} \ddot{a}_{b}\left[(n+2)-\frac{\epsilon}{\left(a_{b} / r_{c}\right)^{\epsilon-2}}\right], \quad k=1 \\
2+\frac{\epsilon(-1)^{\frac{\epsilon}{2}}}{\left(a_{b} / r_{c}\right)^{\epsilon-2}}+a_{b} \ddot{a}_{b}\left[(n+2)+\frac{\epsilon(-1)^{\frac{\epsilon}{2}}}{\left(a_{b} / r_{c}\right)^{\epsilon-2}}\right]>0, \quad k=-1
\end{gathered}
$$

The evolution of the scale factor $a$, the sum of the energy density and pressure $(\rho+p)$ and the energy density $\rho$ during the bounce period for power law corrected model in fractal universe are shown in Figs. 14-16. A barotropic equation of state $p=\gamma \rho$ is assumed for the matter content. The parameters are chosen differently for generating the plots. For scale factor we have chosen $k=G=\Lambda=1, n=2, v_{0}=0.2, r_{c}=10, \epsilon=4$ and $\gamma=-1 / 2$ (both blue and red lines with different initial conditions) and $\gamma=1$ (green line). Here variations have been made both in terms of initial conditions and choice of $\gamma$ to achieve a bouncing solution. However, the plots for $\rho+p$ and $\rho$ were carried out with a different choices of the parameter $\epsilon$, since it plays a crucial role for the satisfaction of NEC. In these two cases, the initial conditions are kept constant. The parameters are chosen for these two cases as $k=-1, G=\Lambda=1, n=2, r_{c}=10, v_{0}=0.2, \gamma=1 / 3$ and $\epsilon=6$ (green line), 10 (red line) and 14 (blue line). it can be observed that the plots show the satisfaction of the NEC.

\section{Discussions and Concluding Remarks}

In this work, the modified field equations in logarithmic and power law versions of entropy corrected models in Einstein's gravity in the background of FRW Universe are studied. After discussing briefly about the Hořava-Lifshitz gravity, we have formulated the modified field equations in logarithmic and 


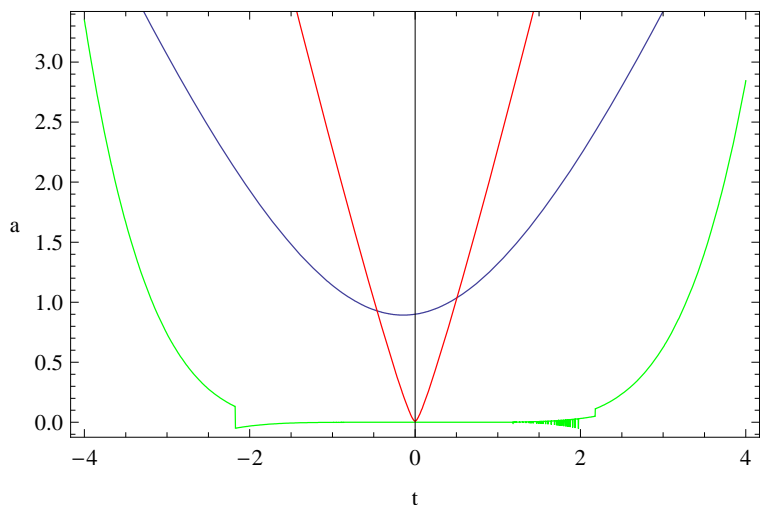

Fig.14

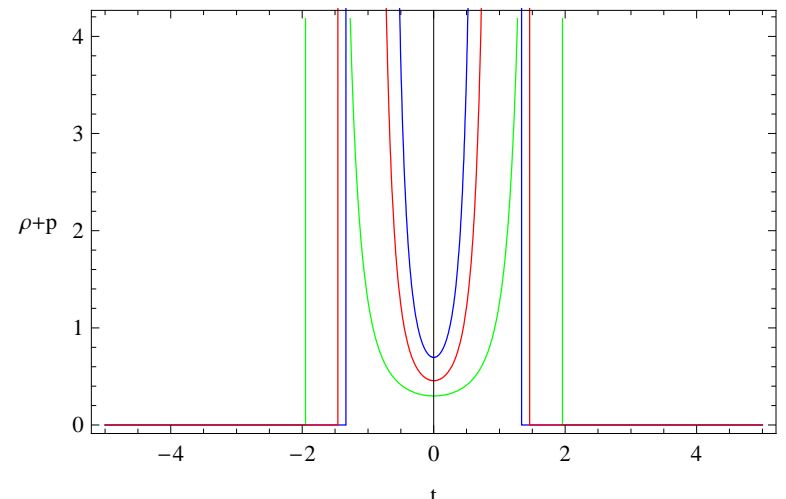

Fig.15

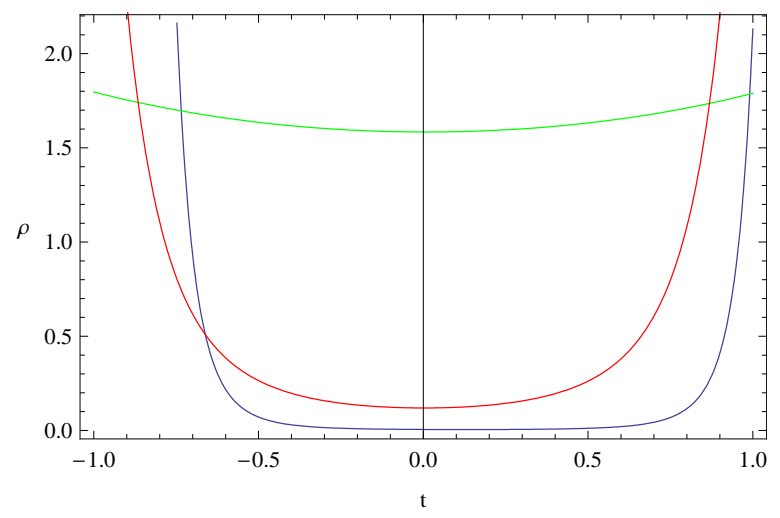

Fig.16

Figs. 14-16 show the dynamical behaviors of the scale factor $a$, the sum of the energy density and pressure $(\rho+p)$ and the energy density $\rho$ during the bounce period for power law corrected model in fractal universe. 
power law versions of entropy corrected models in Hořava-Lifshitz gravity. We have performed the stability analysis of the dynamical system for these models. For logarithmic version of entropy corrected model in Hořava-Lifshitz gravity, we have obtained a stable critical point by assuming $k=-1$, $\Lambda=\alpha^{\prime}=\beta^{\prime}=1, \gamma=1 / 3, \nu=1$. The graph of $a$ vs $H$ in this case is drawn in Fig. 1 and we have found that the critical point is a stable center. For power law version of entropy corrected model in Hořava-Lifshitz gravity, we have obtained a stable critical point by assuming $k=1, \Lambda=\nu=1, \epsilon=2$, $r_{c}=10$ and $\gamma=1 / 3$. The phase space diagram of $a$ vs $H$ is shown in Fig.2 and a stable center is found.

In another section, we have briefly discussed about the fractal universe and later have formulated the modified field equations in logarithmic and power law versions of entropy corrected models in fractal universe. The stability analysis for the dynamical system is then performed for these models in the framework of fractal universe. For logarithmic version of entropy corrected model in fractal universe, we have found the stable critical point for the choice of the parameters $k=1$, $\Lambda=\alpha^{\prime}=\beta^{\prime}=1, n=2, v_{0}=0.2, \omega=0.3$ and $\gamma=0$. From the phase diagram Fig. 3, we have got a stable center. For power law version of entropy corrected model in fractal universe, a stable critical point is obtained by choosing $k=-1, \Lambda=1, \epsilon=4, r_{c}=10, n=2, v_{0}=0.5, \omega=0.6$ and $\gamma=-1 / 2$. The phase space diagram of $a$ vs $H$ has been presented in Fig.4 and a stable center is obtained.

Furthermore, we have analyzed the bouncing scenarios of the universe in Hořava-Lifshitz gravity and fractal model for both logarithmic and power law entropy corrected versions in $k=0,+1,-1$ separately. For different cases, we have examined the validity of the null energy condition (NEC) at the time of bounce. For logarithmic corrected entropy in Hořava-Lifshitz gravity with $k=0$, we have observed that the NEC is violated. Also $\rho_{b}$ attained a minimum value during bounce given by $\left(\rho_{b}\right)_{\min }=-\frac{3 \nu \Lambda}{16 \pi G_{c}}$. So this case has turned out to be unfavorable for bounce. On the other hand, for $k= \pm 1$, the NEC may be satisfied with some conditions provided in equations (59) and (600). Also $\rho_{b}$ may attain maximum value during bounce, which are given in equations (57) and (58). In the case, the evolution of the scale factor $a,(\rho+p)$ and $\rho$ during the bounce period are shown in Figs. 5-7. For viable choices of the parameters involved, the plots shown an oscillating universe with the NEC being satisfied.

For power law corrected entropy in Hořava-Lifshitz gravity with $k=0$, we have observed that the NEC is violated and at the time of bounce, the energy density becomes negative, which shows there is no bounce. But for $k= \pm 1$ with $\epsilon=2 m$, where $m$ is odd, there would be a chance to have bounce. In all other cases, we have unfavorable condition for bounce. In this case, $\rho_{b}$ attains its maximum value. The bouncing solution, the evolution of the scale factor $a,(\rho+p)$ and $\rho$ during the bounce period are shown in Figs. 8-10.

For logarithmic corrected entropy in fractal universe with $k=0$, we have observed that the NEC is satisfied for a negative $n$ and violated otherwise. This case is not favorable for bounce as $\rho_{b}$ becomes negative. But for $k \pm 1$, NEC may be satisfied with some restrictions. The evolution of the scale factor $a,(\rho+p)$ and $\rho$ during the bounce period are shown in Figs. 11-13. The plots clearly show a bouncing solution with the NEC being satisfied.

For power law corrected entropy in fractal universe with $k=0$, we have observed that the NEC is satisfied when $n<-2$. But this solution indicates an unfavorable criteria for a bounce as $\rho_{b}$ becomes negative. But for $k= \pm 1$ with $\epsilon=2 m$, where $m$ is odd, one can attain a bouncing solution. In this case, $\rho_{b}$ attains its maximum value. The validity of NEC depends on some restrictions. The evolution of the scale factor $a,(\rho+p)$ and $\rho$ during the bounce period are shown in Figs. 14-16. From the figures, it can be observed that the plots show the satisfaction of the NEC. 
Acknowledgement: The author TB is thankful to IUCAA, Pune, for their warm hospitality

where part of the work has been done during a visit under the Associateship Programme.

\section{References}

[1] V. Mukhanov, Physical Foundations of Cosmology (Cambridge University Press, Cambridge, UK, 2005).

[2] V. Mukhanov and G. Chibisov, JETP Lett. 33, 532 (1981).

[3] K. Sato, Mon. Not. R. Astron. Soc. 195, 467 (1981).

[4] D. H. Lyth and A. Riotto, Phys. Rep. 314, 1 (1999).

[5] A. Linde, arXiv:1402.0526.

[6] A. Borde and A. Vilenkin, Phys. Rev. Lett. 72 3305, (1994).

[7] M. Novello and S. E. P. Bergliaffa, Phys. Rep. 463, 127 (2008).

[8] V. F. Mukhanov and R. H. Brandenberger, Phys. Rev. Lett. 68, 1969 (1992).

[9] S. D. Odintsov and V. K. Oikonomou, Phys. Rev. D 94, 064022 (2016).

[10] R. C. Tolman, Relativity, Thermodynamics and Cosmology, Clarendon, Oxford (1934).

[11] L. Xu and H. Liu, Int. J. Mod. Phys. D 14, 1947 (2005).

[12] S. Nojiri and S. D. Odintsov, Mod. Phys. Lett. A 29, 1450211 (2014).

[13] V. F. Antunes and M. Novello, Gravitation and Cosmology 22, 1 (2016).

[14] J. Gegenberg, S. Rahmati and S. S. Seahra, Phys. Rev. D 95, 043509 (2017).

[15] Y. F. Cai, A. Marcian, D. G. Wang and E. W. Ewing, Universe 3, 1 (2017).

[16] S. Carloni, P. K. S. Dunsby and D. Solomons, Class. Quantum Grav. 23, 1913 (2006).

[17] C. Barragan, G. J. Olmo and H. S. Alepuz, Phys. Rev. D 80, 024016 (2009).

[18] N. Paul, S. N. Chakrabarty and K. Bhattacharya, JCAP 10, 009 (2014).

[19] M. Ghanaatian and F. Milani, Gen. Rel. Grav. 46, 1789 (2014).

[20] M. K. Fard, Int. J. Theor. Phys. 54, 1938 (2015).

[21] S. D. Odintsov and V. K. Oikonomou, Phys. Rev. D 91, 064036 (2015).

[22] S. D. Odintsov and V. K. Oikonomou, Phys. Rev. D 92, 024016 (2015).

[23] Y. F. Cai, S. H. Chen, J. B. Dent, S. Dutta and E. N. Saridakis, Class. Quantum Grav. 28, 215011 (2011).

[24] A. V. Astashenok, Astrophys. Space Sci. 351, 377 (2014). 
[25] M. Bojowald, R. Maartens and P. Singh, Phys. Rev. D 70, 083517 (2004).

[26] P. Singh, K. Vandersloot, and G. V. Vereshchagin, Phys. Rev. D 74, 043510 (2006).

[27] J. de Haro, J. Cosmol. Astropart. Phys. 11, 037 (2012).

[28] P. J. Steinhardt and N. Turok, Phys. Rev. D 65, 126003 (2002).

[29] P. J. Steinhardt and N. Turok, Science 296, 1436 (2002).

[30] Y. S. Piao, Phys. Rev. D 70, 101302 (2004).

[31] Y. S. Piao and Y. Z. Zhang, Nucl. Phys. B 725, 265 (2005).

[32] T. Singh, R. Chaubey and A. Singh, Eur. Phys. J. Plus 130, 31 (2015).

[33] K. Bamba, A. N. Makarenko, A. N. Myagky, S. D. Odintsov, Phys. Lett. B 732, 349 (2014).

[34] V. K. Oikonomou, Phys. Rev. D 92, 124027 (2015).

[35] I. Terrucha, D. Vernieri and J. P. S. Lemos, Annals Phys. 404, 39 (2019).

[36] A. Escofet and E. Elizalde, Mod. Phys. Lett. A 31, 1650108 (2016).

[37] D. N. Page, Class. Quant. Grav. 1, 417 (1984).

[38] S. D. Sadatian, Int. J. Theor. Phys. 53, 675 (2014).

[39] A. Salehi, Phys. Rev. D 94, 123519 (2016).

[40] S. Chattopadhyay, Int. J. Geom. Meth. Mod. Phys. 14, 1750181 (2017).

[41] T. Bandyopadhyay and U. Debnath, Can. J. Phys. 97, 286 (2019).

[42] S. Colin and N. Pinto-Neto, Phys. Rev. D 96, 063502 (2017).

[43] M. Arab and A. Khodam-Mohammadi, Eur. Phys. J. C 78, 243 (2018).

[44] A. N. Makarenko and A. N. Myagky, Int. J. Geom. Meth. Mod. Phys. 15, 1850096 (2018).

[45] S. F. Bramberger and J. -Luc Lehners, Phys. Rev. D 99, 123523 (2019).

[46] A. A. Aly, Pramana 92, 34 (2019).

[47] R. B. Mann and S. N. Solodukhin, Nucl. Phys. B 523, 293 (1998).

[48] K. A. Miessner, Class. Quantum Grav. 215245 (2004).

[49] A. Chatterjee and P. Majumder, Phys. Rev. Lett. 92141301 (2004).

[50] S. K. Modak, Phys. Lett. B 671167 (2009).

[51] H. M. Sadjadi and M. Jamil, Europhys. Lett. 9269001 (2010).

[52] R. G. Cai, L. M. Cao and Y.P. Hu, JHEP 0808, 090 (2008).

[53] M. Akbar and R. G. Cai, Phys. Rev. D 7, 084003 (2007).

[54] S. Das, S. Shankaranarayanan and S. Sur, Phys. Rev. D 77, 064013 (2008). 
[55] N. Radicella and D. Pavon, Phys. Lett. B 691, 121 (2010).

[56] A. Sheykhi and M. Jamil, Gen. Relativ. Gravit. 43, 2661 (2011).

[57] P. Horava, Phys. Rev. D 79, 084008 (2009).

[58] P. Horava, JHEP 0903, 020 (2009).

[59] P. Horava, Phys. Rev. Lett. 102, 161301 (2009).

[60] R. L. Arnowitt, S. Deser and C. W. Misner, In: L. Witten (ed.) Gravitation: an Introduction to Current Research, pp. 227265 (2004). Wiley 1962, Chap. 7.

[61] S. Nojiri and S. D. Odintsov, Phys. Rep. 505, 59 (2011).

[62] W. Hao, Commun. Theor. Phys. 52, 743 (2009).

[63] G. Calcagni, JHEP 1003, 120 (2010).

[64] G. Calcagni, Phys. Rev. Lett. 104 (2010) 251301. 